\title{
¿Por qué los pacientes no retiran todos los medicamentos y productos sanitarios que les son prescritos por el médico?
}

\section{Rosa Prats Mas ${ }^{1}$, Jaime Botella Ripoll2, Daniel Dols Alonso ${ }^{3}$, Vicente Baixauli Fernández ${ }^{4}$, María Teresa Climent Catalá ${ }^{5}$}

1. Doctora en Farmacia. Farmacéutica comunitaria en Dénia (Alicante). 2. Farmacéutico comunitario en Palmeras (Sueca - Valencia). 3. Farmacéutico comunitario en Castellón. 4. Doctor en Farmacia. Farmacéutico comunitario en Mislata (Valencia). 5. Doctora en Farmacia. Farmacéutica comunitaria en L'Olleria (Valencia).

\section{PALABRAS CLAVE}

Farmacia comunitaria, receta electrónica, adherencia al tratamieto, dispensación

\section{ABREVIATURAS}

COF: Colegios Oficiales de Farmacéuticos

CV: Comunidad Valenciana FC: Farmacia comunitaria GAIA: Gestor integral para la prestación farmacéutica de la Conselleria de Sanitat de la Generalitat Valenciana. HTV: Hoja de Tratamiento Vigente MPRE: Módulo de prescripción MPS: Medicamento o producto sanitario

PRM: Problema relacionado con los medicamentos

RELE: Receta electrónica

SGF: Sistema de Gestión

Farmacéutica

SICOF: Sistema de Información Colegial

SIP: Tarjeta sanitaria individual del paciente

\section{KEYWORDS}

Community pharmacy, electronic prescription, medication adherence, dispensation

\section{RESUMEN}

Introducción: la e-receta finalizó su implantación en la Comunidad Valenciana (CV) en septiembre de 2013, observándose que el paciente no retiraba parte de los medicamentos que tenía prescritos, por lo que se diseña un estudio para estudiar las diversas incidencias que pudieran ser la causa.

Objetivos: analizar y cuantificar las incidencias detectadas en la dispensación de las e-receta prescritas a los pacientes en farmacia comunitaria de la CV.

Métodos: estudio observacional, transversal, prospectivo multicéntrico de base poblacional de las incidencias detectadas en las dispensaciones de las e-receta. Población diana: pacientes mayores de 18 años o cuidadores.

Resultados: se registraron 22.983 solicitudes de medicamentos o productos sanitarios (MPS) correspondientes a 6.621 pacientes. De los MPS disponibles para ser dispensados se rechazaron un $12,0 \%$

- Las discrepancias entre lo que el médico prescribió y el paciente entendió supusieron el $19,0 \%$ de los rechazos.

- Las distintas razones relacionadas con el incumplimiento fueron el 32,3 \% de los medicamentos rechazados (MR).

- En aquellos medicamentos en los que el paciente debía pagar una aportación el rechazo fue ligeramente mayor (12,2\%) que en el caso de los que no.

Conclusiones: la dispensación de MPS prescritos a través de la e-receta permite identificar problemas relacionados con medicamentos (PRM) que inciden sobre la falta de adherencia y las discrepancias con lo prescrito en la receta electrónica.

Why do patients not withdraw all the medicines and health products prescribed to them by the doctor?

\section{ABSTRACT}

Introduction: The e-receipt finished its implantation in the Valencian Community (CV) in September 2013, observing that the patient did not withdraw part of the medicines prescribed, so a study is designed to find out the various incidents that could be the cause.

Objectives: To analyze and quantify the incidences detected in the dispensation of the e-recipes prescribed to patients in community pharmacy (FC) de la CV.

Methods: Observational, cross-sectional, prospective multicenter population-based study of the incidences detected in the dispensations of the e-recipes. The target population: patients over 18 years of age, or caregivers.

Results: 22,983 requests for medicines or health products (HP) were recorded for 6,621 patients. Of the medicines or HP available for dispensing, 12.0\% were rejected.

- Discrepancies between what the doctor prescribed and what the patient understood accounted for $19.0 \%$ of refusals.

- The different reasons related to non-compliance were $32.3 \%$ of medicines or health products (HP).

- In those medicines where the patient had to pay a contribution, the rejection was slightly higher (12.2\%) than in the case of those who did not.

Conclusions: The dispensing of prescribed medicines/HP through the e-prescription makes it possible to identify drug related problem DRPs that affect the lack of adherence and discrepancies with what is prescribed in the electronic prescription. 


\section{Introduccion}

En el año 2002, en la Comunidad Valenciana (CV), se produce un profundo e importante cambio en el modelo de receta médica al pasarse de la receta manuscrita a la receta informatizada. Este cambio se lleva a cabo a través del Gestor Integral de la Prestación Farmacéutica (GAIA) (1) que aporta una serie de ventajas que se pueden concretar en la minimización de errores en la dispensación, la incorporación de información al paciente para la correcta administración y duración de tratamiento y la creación de un historial farmacoterapéutico del paciente, que puede ser consultado en cualquier centro de salud dentro de la CV (2).

En 2004 se da un paso más y se establecen las bases de lo que será la receta eléctrónica (RELE), tal como la conocemos en la actualidad (3). El sistema de RELE permite llevar de forma automatizada las funciones administrativas y de prescripción necesarias para la dispensación de medicamentos (4).

En 2007 se dio el empujón definitivo para su implantación al firmarse un convenio de colaboración entre los Colegios Oficiales de Farmacéuticos (COF) de la CV y la Conselleria de Sanitat de la CV (5).

Como consecuencia, la receta electrónica comenzó a implantase en la CV en marzo de 2008 (6) y finalizó en septiembre de 2013 (7-9), constando de tres sistemas de información:

- Módulo de prescripción (MPRE), a través del cual los facultativos determinan los tratamientos farmacológicos de los pacientes (incluido en GAIA).

- Sistema de Gestión Farmacéutica (SGF), que es el sistema de gestión propio de cada farmacia comunitaria $(\mathrm{FC})$, a través del cual se realiza la dispensación.

- SICOF es el sistema de información de los COF de la CV y que se sitúa entre GAIA y los SGF.

El proceso de la RELE es sencillo. El médico prescribe al paciente la medicación que este precisa en MPRE y hace entrega al paciente de una Hoja de Tratamiento Vigente (HTV) en la que figuran, entre otros datos, todos los medicamentos prescritos activos, dosis y posología, pauta de administración y duración del tratamiento, así como un calendario con las fechas de dispensación de los medicamen- tos prescritos que le permita saber al paciente cuando debe ir a la farmacia comunitaria (FC) a recogerlos. Para obtener la medicación prescrita y poder realizar la dispensación de dichos medicamentos, el farmacéutico debe acceder a través del SICOF al GAIA identificándose mediante unos certificados electrónicos y debe introducir, por un lado, el número de la tarjeta sanitaria individual del paciente (SIP), que éste le debe proporcionar y que el farmacéutico captura leyendo su banda magnética a través de su SGF y, por otro, el código de barras que figura en la HTV que también debe aportar el paciente en el momento de la dispensación. Una vez introducida esta información, el farmacéutico podía visualizar en la pantalla de su SGF los medicamentos prescritos y aquellos disponibles para ser dispensados.

Durante los primeros meses tras la implantación de la RELE se pudo observar un elevado número de incidencias, que se fueron solventando poco a poco. Pero una vez transcurrido un tiempo prudencial se observó que el paciente no retiraba parte de los medicamentos que tenía prescritos, planteándose la hipótesis de si este hecho pudiera estar relacionado, por un lado, con una falta de adherencia del paciente a la medicación, tanto voluntaria como involuntaria, $\mathrm{y}$, por otro, con errores derivados del manejo de la MPRE o por el uso de las HTV por los pacientes. Para estudiar estas incidencias, sus posibles causas y sus posibles soluciones, desde la delegación de la CV de la Sociedad Española de Farmacia Familiar y Comunitaria (SEFAC) se diseñó un estudio de investigación.

\section{Objetivos}

\section{Objetivo principal}

Analizar las incidencias en la dispensación de las recetas electrónicas prescritas a los pacientes en farmacias comunitarias de la Comunidad Valenciana.

\section{Objetivos secundarios}

- Cuantificar las incidencias registradas.

- Describir la población estudiada según edad y sexo.

- Analizar si existe alguna relación causal entre el rechazo de ciertas dispensaciones y la necesidad de aportación económica por el paciente.

\section{Metodología}

Estudio observacional, transversal, prospectivo multicéntrico de base poblacional.

El objeto del estudio fueron las incidencias detectadas en las dispensaciones realizadas a partir de las prescripciones GAIA, emitidas como recetas electrónicas para su dispensación en las farmacias comunitarias de la CV participantes en el estudio. $\mathrm{Su}$ duración fue de tres meses (desde enero a marzo de 2016).

La población de estudio fueron los pacientes que acudieron a las FC participantes en el estudio a los que se les dispensasen los medicamentos prescritos en la receta electrónica por los facultativos dependientes de la Conselleria de Sanitat.

\section{Criterios de inclusión}

Pacientes mayores de 18 años que, bien directamente o a través de sus cuidadores, acudieron a la farmacia, en su horario habitual de apertura, con recetas prescritas a través de RELE de la CV.

\section{Criterios de exclusión}

Pacientes que no cumplen los criterios de inclusión.

\section{Aleatorización}

Durante el periodo de estudio cada participante dividió su jornada laboral en franjas horarias iguales y seleccionó de forma aleatoria, cada día, en cual iba a registrar pacientes. Los pacientes que se registraban se elegían antes de que entraran en la farmacia; por tanto, no se podía saber quiénes iban a ser antes de ser seleccionados.

\section{Variables}

Los datos recogidos en las dispensaciones se recogen en una base de datos ad hoc realizada en ACCESS ${ }^{\circledR}$, donde se recogieron: fecha, sexo (hombre/mujer), edad (años), aportación (si el paciente tiene que abonar una aportación económica por los medicamentos o no), código nacional de los medicamentos o productos 
sanitarios disponibles para su dispensación, códigos nacionales de los medicamentos o productos sanitarios que se rechazan y el motivo por el que se rechazan de acuerdo con los ítems de la tabla 1. También se registran los medicamentos que el paciente demanda pero que no están disponibles para su dispensación en el momento en que acude el paciente a la FC (tabla 1).

\section{Farmacias y farmacéuticos} comunitarios participantes

Para la realización del estudio se contó con la participación libre, voluntaria y altruista de 14 farmacéuticos comunitarios asociados a SEFAC ejercientes en $14 \mathrm{FC}$ de las tres provincias de la CV. En el apartado Farmacéuticos participantes en el estudio figuran los nombres de los farmacéuticos comunitarios y la distribución de las FC por provincias.

\section{Pilotaje}

Se realizó un estudio piloto previo en enero de 2014. Los resultados fueron presentados en el Congreso $\mathrm{Na}$ cional de Farmacéuticos Comunitarios celebrado en 2014 en Málaga (10-12).
El procedimiento para la obtención de datos es el de dispensación habitual con receta electrónica:

1. Paciente (o cuidador) acude a la FC con su tarjeta sanitaria individual y su HTV.

2. En el SFG se captura la banda magnética de la tarjeta y el código de barras de la HTV.

3. Se visualizan en pantalla los medicamentos que el paciente tiene prescritos y de ellos cuáles están disponibles para su dispensación en ese momento. En la CV los medicamentos están disponibles para la dispensación hasta 10 o 14 días después de la fecha de recogida que indica la HTV, si bien existe una utilidad que permite "replanificar" si el medicamento no fue retirado en dicho plazo.

4. El paciente decide qué medicamentos quiere que se le dispensen.

5. Si el paciente refiere que necesita algún medicamento que no está disponible para dispensar, se valora si es FMP o FMNP. Si se puede replanificar para poder disponer de ellos, se replanifica.

6. Se procede a dispensar los medicamentos indicados. Si el paciente rechaza algún medicamento se registra la causa de rechazo y se le aconse- ja consultar con el médico si se considera necesario. En los pacientes no adherentes se intervino para intentar reconducirlos hacia el cumplimiento mediante educación sanitaria en el uso racional del medicamento y la importancia de la adherencia al tratamiento.

\section{Análisis de datos}

Los datos registrados en la base de datos, una vez depurados, se analizaron estadísticamente con Microsoft Excel $^{\circledR}$. Para comparar variables categóricas se utilizó la prueba chi cuadrado y para las numéricas la prueba t de Student. Se consideró estadísticamente significativo $\mathrm{p}<0,05$.

\section{Limitaciones}

La principal limitación del estudio es la relacionada con la veracidad de las afirmaciones del paciente.

\section{Aspectos éticos}

Dado que el procedimiento utilizado fue el que se realiza en la práctica clínica habitual cada vez que se dispensa un medicamento, y que no

Tabla 1 Causas de rechazo y reclamación para la recogida de la medicación con receta electrónica

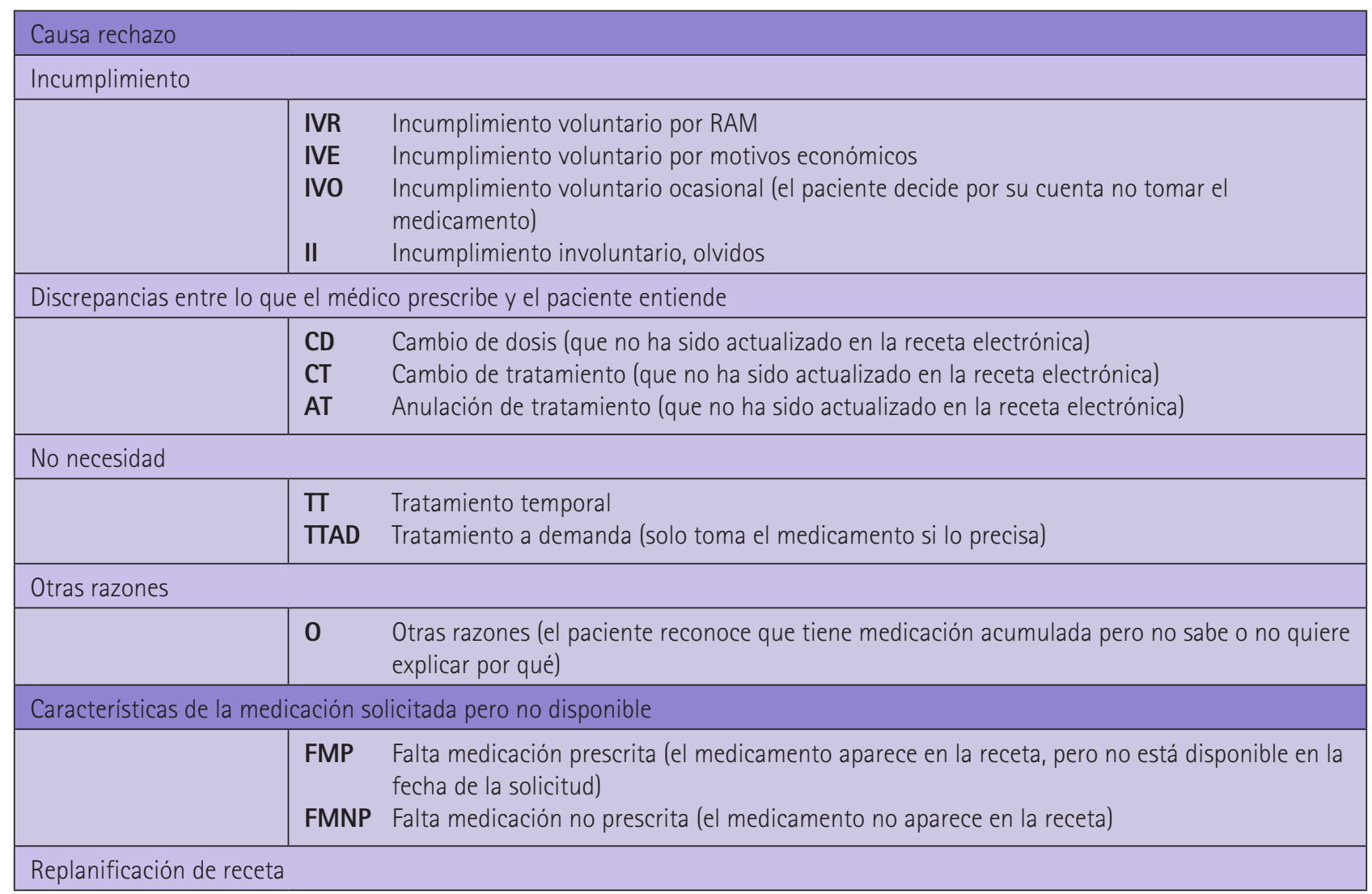


se obtuvieron datos identificativos de los pacientes, no se consideró necesaria la petición de consentimiento informado al paciente.

La Agencia Española de Medicamentos y Productos Sanitarios (AEMPS) clasificó el estudio como EPA-OD con el código: SEF-IRE-2015-01, y fue aprobado por el Comité Ético Autonómico de Estudios Clínicos de Medicamentos y Productos Sanitarios de la CV.

Ninguno de los investigadores manifestó tener conflictos éticos y de interés para la realización del estudio.

\section{Resultados}

Se registraron 22.983 solicitudes de medicamentos o productos sanitarios (MPS) realizadas por 6.621 pacientes; $3.680(55,6 \%)$ mujeres. La edad media fue de 66,4 años (DE 15,6); 4.071 $(61,5 \%)$ fueron mayores de 65 años.

El promedio de medicamentos dispensados a mayores de 65 años fue de 3,80 y a menores de 65 años fue 2,98 $(\mathrm{p}<0,001)$.
Estudio de las incidencias detectadas

De las 22.983 solicitudes de MPS realizadas por los pacientes, 22.899 (99,6 \%) estaban disponibles en la RELE para su dispensación en el momento de la solicitud y $84(0,4 \%)$ no.

Causas de rechazo de medicamentos o productos sanitarios en dispensación

De los MPS disponibles para ser dispensados los pacientes rechazaron la dispensación de 2.754 (12,0 \%). Las causas fueron las siguientes:

- Las discrepancias entre lo que el médico prescribió y el paciente entendió supusieron el 2,3\% de los disponibles para dispensar y el 19,0 \% de los rechazados.

- Las distintas razones relacionadas con el incumplimiento fueron el $3,9 \%$ de los disponibles para dispensar y el 32,3 \% de los rechazados, siendo el rechazo por incumplimiento voluntario $(21,9 \%)$ superior al involuntario (10,4\%).
- El rechazo por realmente no necesitar el medicamento fue del 2,5\% de los medicamentos disponibles para dispensar y el 21,2\% de los medicamentos rechazados.

- Y el rechazo por otras razones no aclaradas fue del 3,3 \% de los medicamentos disponibles para dispensar y el 27,6 \% de los medicamentos rechazados.

En la tabla 2 se muestra el detalle de las causas de rechazo y reclamación de los medicamentos solicitados.

\section{Rechazo en función de la} aportación

El 23,0 \% de los medicamentos solicitados estaban exentos de aportación y el 77,0 \% restante requirieron del pago de algún importe. En aquellos medicamentos en los que el paciente debía pagar una aportación el rechazo fue ligeramente mayor (12,2\%) que en el caso de los que no necesitaban aportación $(11,2 \%)(p=0,04)$.

Tabla 2 Detalle de los rechazos y reclamaciones de medicamentos y productos sanitarios según su causa. Porcentajes calculados sobre el total de rechazos o reclamaciones y sobre el total de productos demandados

\begin{tabular}{|c|c|c|c|c|}
\hline \multicolumn{2}{|c|}{ Medicamentos rechazados } & $\mathrm{n}$ & $\%$ Rechazo & $\%$ Demanda \\
\hline \multicolumn{2}{|c|}{ Discrepancias } & 522 & $19,0 \%$ & $2,3 \%$ \\
\hline AT & Anulación de tratamiento & 249 & $9,0 \%$ & $1,1 \%$ \\
\hline CD & Cambio de dosis & 200 & $7,3 \%$ & $0,9 \%$ \\
\hline CT & Cambio de tratamiento & 73 & $2,7 \%$ & $0,3 \%$ \\
\hline \multicolumn{2}{|c|}{ Incumplimiento } & 889 & $32,3 \%$ & $3,9 \%$ \\
\hline II & Incumplimiento Involuntario, olvidos & 286 & $10,4 \%$ & $1,2 \%$ \\
\hline IVE & Incumplimiento voluntario por motivos económicos & 46 & $1,7 \%$ & $0,2 \%$ \\
\hline IVO & Incumplimiento voluntario ocasional & 510 & $18,5 \%$ & $2,2 \%$ \\
\hline IVR & Incumplimiento voluntario por RAM & 47 & $1,7 \%$ & $0,2 \%$ \\
\hline \multicolumn{2}{|c|}{ No Necesidad } & 583 & $21,2 \%$ & $2,5 \%$ \\
\hline TAD & Tratamiento a demanda & 514 & $18,7 \%$ & $2,2 \%$ \\
\hline$\pi$ & Tratamiento temporal & 69 & $2,5 \%$ & $0,3 \%$ \\
\hline \multicolumn{2}{|c|}{ Otras razones } & 760 & $27,6 \%$ & $3,3 \%$ \\
\hline 0 & Otras razones & 760 & $27,6 \%$ & $3,3 \%$ \\
\hline \multicolumn{2}{|c|}{ Total de medicamentos rechazados } & 2.754 & $100 \%$ & $11,98 \%$ \\
\hline \multicolumn{2}{|c|}{ Medicamentos no rechazados } & 20.145 & & $87,65 \%$ \\
\hline \multicolumn{2}{|c|}{$\begin{array}{l}\text { Medicamentos reclamados } \\
\text { (medicamentos solicitados no disponibles) }\end{array}$} & $\mathrm{n}$ & \% Reclamación & $\%$ Demanda \\
\hline \multicolumn{2}{|c|}{ FMNP Falta medicación no prescrita } & 24 & $28,6 \%$ & $0,1 \%$ \\
\hline & & 60 & $71,4 \%$ & $0,3 \%$ \\
\hline \multicolumn{2}{|c|}{ Total de medicamentos reclamados } & 84 & $100 \%$ & $0,37 \%$ \\
\hline \multicolumn{2}{|c|}{ Total medicamentos solicitados } & 22.983 & & $100 \%$ \\
\hline
\end{tabular}




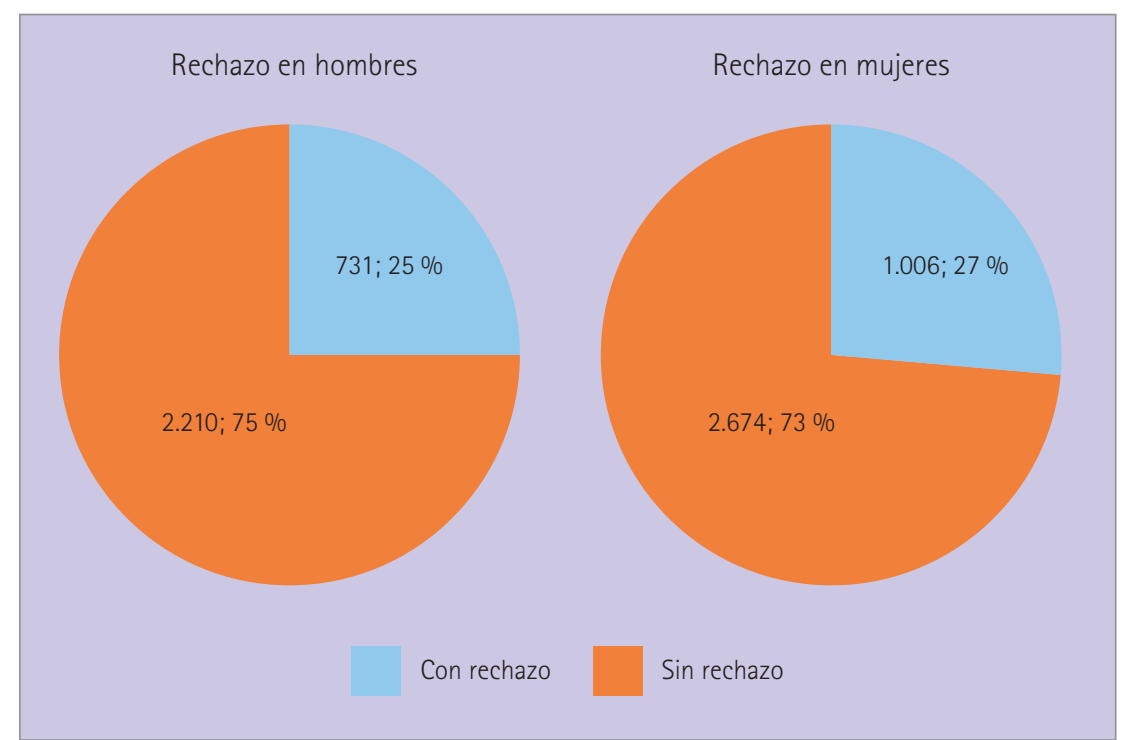

Figura 1 Porcentaje de pacientes que rechazan algún MPS en función del sexo $(p=0,02)$

Descripción de los pacientes que rechazan algún medicamento

$1.737(26,2 \%)$ pacientes rechazaron algún medicamento. En la figura 1 puede verse el rechazo según el sexo, y en la figura 2 el rechazo en función de la edad.

Descripción de los pacientes a los que les falta algún medicamento

77 pacientes reclamaron 84 medicamentos que no estaban disponibles en RELE para su dispensación en ese momento. La edad media de los pacientes fue de 68,4 años (DE 13,6). Fueron el 1,1 \% de los hombres y el $1,3 \%$ de las mujeres $(\mathrm{p}=0,465)$.

\section{Discusion}

Los resultados del estudio indican que el paciente que acude a la farmacia a que se le dispensen los medicamentos retira en la mayoría de los casos los medicamentos prescritos por los facultativos médicos, rechazándose un $12,0 \%$ de ellos y demandándose un $0,4 \%$ que no están disponibles en ese momento para su dispensación. En definitiva, un $12.4 \%$ de incidencias sobre las cuales se debería intervenir.

El análisis de las causas del rechazo indica que existen algunos problemas relacionados con los medicamentos (PRM). El más frecuente y que constituye la tercera parte de

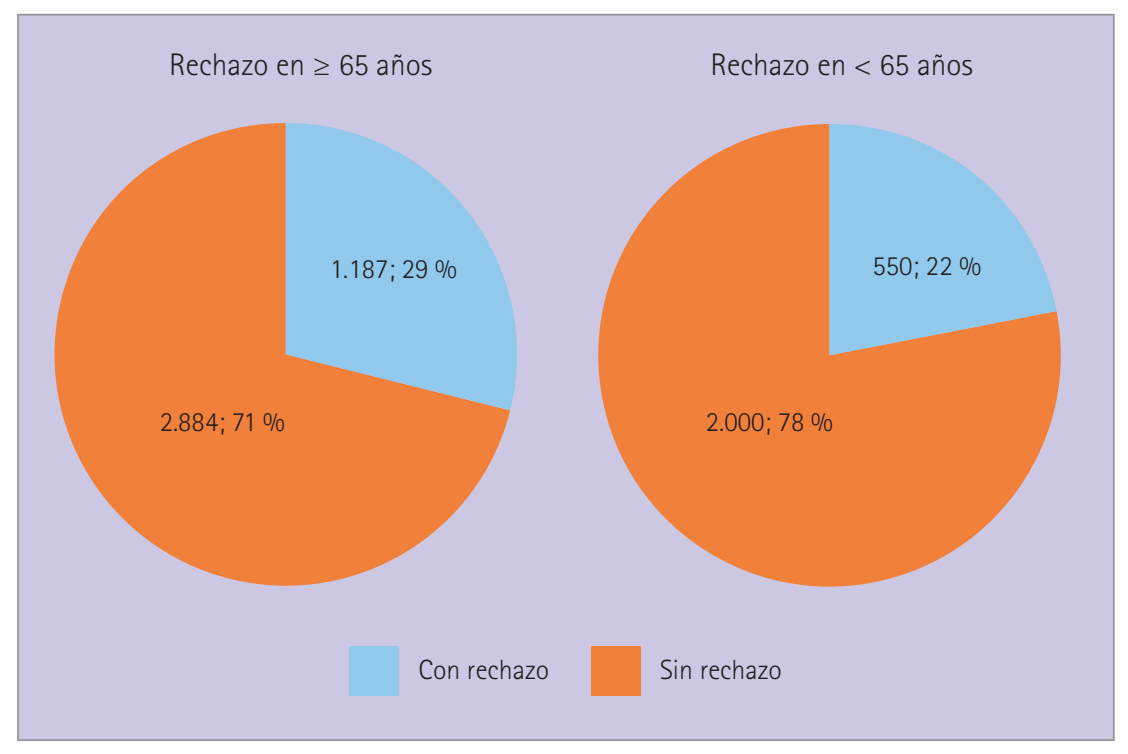

Figura 2 Porcentaje de pacientes que rechazan algún MPS según la edad $(p<0,01)$ los rechazos es el incumplimiento terapéutico (y de ellos, la tercera parte se debe a olvidos), seguido de discrepancias entre el tratamiento prescrito por escrito y el prescrito de forma oral, según el paciente. En cualquier caso, el paciente rechaza y no utiliza algunos medicamentos porque según el propio paciente, el médico le ha indicado de forma verbal unas instrucciones que no coinciden con las que figuran escritas en la receta, y ante la duda, opta por no utilizarlo.

Ante ambas causas de rechazo, en las que el paciente no retira su medicación, el farmacéutico comunitario podría intervenir intentando mejorar la adherencia al tratamiento prescrito.

Pero también hay una parte importante, 3,3\% de los medicamentos rechazados, en la que no hemos podido averiguar la razón del rechazo, y que podría aumentar los ya comentados o enmascarar otros.

Sólo estaría plenamente justificado el rechazo de los medicamentos en el $21,2 \%$ de las ocasiones, ya que el paciente no necesita los medicamentos bien porque tiene de sobra (se prescribieron "a demanda"), que es la causa más habitual, o bien porque el medicamento se prescribió durante una temporada y ya no lo necesita. En estos casos el médico tiende a prescribir una cantidad suficiente para que, probablemente con buen criterio, al paciente no le falte su medicación. Caso muy frecuente en prescripciones de analgésicos o broncodilatadores de rescate, y también con el acenocumarol, pero por otras razones.

En relación con la edad se puede pensar que los pacientes mayores rechazan más medicamentos debido a que la media de medicamentos que utilizan es mayor pero no es así, porque los mayores de 65 años rechazan el 12,2 \% de sus medicamentos y los menores de 65 años rechazan el $11,5 \%(p=0,13)$.

En el estudio se ha detectado que un 3,9\% de MPS no se recogen por falta de adherencia. Esto supone que el 26,2 \% de los pacientes no son adherentes a al menos uno de sus medicamentos. Este valor se queda muy por debajo del $50 \%$ que estima la OMS (13) y del 50,1 \% encontrado en 2016 por la Encuesta (14) sobre adherencia terapéutica en España realizada por el Gabinete de Sociología y Comunicación promovida por Farmaindustria. Otros estudios obtienen 
valores muy distintos en función de la patología estudiada y la metodología empleada (15-20). La respuesta más plausible para esta diferencia es que muchos pacientes retiran los medicamentos, pero luego no los toman, por las razones que sean. Esta diferencia es importante porque nos demuestra que es muy difícil estimar la adherencia en función de la retirada o no de los medicamentos a través de la receta electrónica, y que la adherencia por un lado se subestima, ya que hay pacientes que retiran los medicamentos y luego no los toman, pero por otro lado se sobreestima si no se pregunta la causa del rechazo y solo se atribuye a una falta de adherencia la no recogida de los medicamentos, cuando, como hemos visto, solo se podría atribuir alrededor de la tercera parte de los rechazos.

El estudio más parecido al realizado es el de Piñeiro-Abad et al (21), llevado a cabo en una farmacia gallega, con la receta electrónica y un diseño similar. Obtienen un rechazo real del 29,6\%, más del doble que el obtenido en este $(12,0 \%)$. Las razones que pueden explicar esta diferencia son varias. Piñeiro-Abad et al se realizó en una única farmacia sita en un centro comercial, mientras que este estudio se ha realizado en 14 farmacias de las tres provincias de la CV, preferentemente farmacias de barrio. La tipología de los pacientes puede ser muy distinta. Otro factor que también puede influir es la ventana de disponibilidad de la RELE gallega, que es de 30 días mientras que en la CV es de 10. Estas dos razones creemos que pueden aumentar el rechazo en el estudio de Piñeiro-Abad et al.

Una de las posibles razones del rechazo de medicamentos, que genera incumplimiento, puede ser la económica. Este es un tema muy delicado de investigar porque pocas veces el paciente reconoce que no lo retira porque lo tiene que pagar y no lo quiere/ puede pagar. Hemos encontrado un rechazo ligeramente mayor, pero significativo, en los medicamentos que requerian satisfacer una aportación para su dispensación. Un estudio (22) realizado en la CV con pacientes dados de alta de hospitales concluyó que la adherencia no se veía influida por el copago en medicamentos baratos, pero sí lo es en los caros.

En cuanto a la otra razón de rechazo, las discrepancias con las ins- trucciones escritas del médico, hay pocos estudios hechos en farmacias. El más parecido a éste en cuanto a diseño, el de Piñeiro-Abad et al, se centra más en el cumplimiento y no estudia en profundidad este aspecto. Se limita a decir que el rechazo por "incidencia en prescripción" fue el 10,6\% de las recetas no retiradas, que corresponden al 3,6 \% de las recetas disponibles. En nuestro caso es un $19,0 \%$ y un 2,3 \% respectivamente. Estos datos, aunque parecidos, no son directamente comparables porque la categorización de las causas de rechazo no es la misma en los dos estudios.

Un estudio en el que se median desde la FC los conocimientos de los pacientes sobre los medicamentos dispensados fue el D-Valor (23-27). En este estudio se investigaron los conocimientos del paciente en el momento de la dispensación de determinados medicamentos. Los resultados del $D$-Valor indicaron que el 5,9\% de los pacientes desconocía la posología de su medicamento, el 4,9\% no conocía la indicación, el 10,5 \% la forma de uso y el 40,2 \% no conocía la duración del tratamiento. Estos resultados tampoco son comparables con nuestro estudio, ya que no conocer la posología no implica no retirar la medicación. Del mismo modo, no retirar la medicación no implica ser consciente de que no se conoce la posología. Pero estos resultados indican que hay un problema de desconocimiento en los pacientes.

En 10 farmacias de Toledo (28) se investigó el conocimiento de los pacientes de la posología prescrita de los antibióticos. Esto fue antes de la RELE, por tanto, no todos, solo el $33 \%$, tenian instrucciones escritas del médico. Al preguntarles por la pauta prescrita un 19,2\% respondió erróneamente. Este dato es bastante superior al encontrado en el $D$-Valor $\mathrm{y}$ en este estudio. Una diferencia importante es que en dicho estudio solo se estudiaron los antibióticos que no son medicamentos de uso crónico y suelen tener pautas más complejas, lo cual puede justificar el mayor desconocimiento.

Otro estudio (29) más reciente realizado solo en una farmacia y también con antibióticos halló que el $53,2 \%$ de la población estudiada no tenía un conocimiento que le permitiera asegurar un correcto proceso de uso del antibiótico que utilizaba.
Shivangini Sing et al (30) estudiaron, en consultas externas de un hospital, el conocimiento de pacientes diabéticos y lo relacionaron con el nivel cultural sanitario del paciente, descubriendo que el 22,6\% de ellos no conocía la frecuencia de las tomas y que el 28,0 \% no conocía el número de unidades por toma. Además, establecieron una relación directa y significativa entre la cultura sanitaria del paciente y sus conocimientos. En otro estudio (31) se investigó la capacidad de entender el etiquetado de los medicamentos. Solo el 34,7\% entendía tome dos tabletas por vía oral dos veces al día y estaba muy relacionado con la cultura sanitaria del paciente. Sin embargo, al no haber recogido datos de esta naturaleza en los pacientes del estudio no podemos cotejar estos resultados.

La receta electrónica ha demostrado ser una herramienta útil para medir el cumplimiento terapéutico en algunas patologías (32). Sin embargo, los resultados obtenidos en este estudio no lo corroboran como se ha indicado anteriormente. El rechazo a recoger medicación disponible en la receta electrónica permitió detectar problemas de adherencia y de discrepancias con las prescripciones establecidas en la receta por el médico.

En el momento de la dispensación, el farmacéutico comunitario con pocas preguntas y en muy poco tiempo puede detectar PRM e intentar solucionarlos y está demostrado que esta actitud mejora la adherencia (33).

No hemos medido los conocimientos sanitarios de los pacientes, pero parece bastante obvio que nuestra intervención deberá adaptarse a las creencias y conocimientos de los pacientes porque no todos tienen la misma cultura sanitaria.

La receta electrónica es una buena herramienta para mejorar el proceso de dispensación y estudio de posible adherencia.

\section{Conclusiones}

La dispensación de MPS prescritos a través de la receta electrónica permite identificar PRM que inciden sobre la falta de adherencia al tratamiento de los pacientes y discrepancias con lo prescrito en la receta electrónica. 
El 19,0 \% de los MPS que se rechazan lo son por discrepancias entre lo prescrito y lo que el paciente ha entendido, el 32,3\% por incumplimiento y el $27,6 \%$ por otras razones. El 21,2 \% de los MPS rechazados lo son porque han sido prescritos como tratamiento a demanda o temporal y, por tanto, no son necesarios.

\section{Farmacéuticos participantes en el estudio}

\begin{tabular}{|l|l|}
\hline Antonio Herrero & Valencia \\
\hline Cristina Aparicio & Valencia \\
\hline Daniel Dols & Castellón \\
\hline Edelmira Córcoles & Alicante \\
\hline Fernando Mud & Alicante \\
\hline Gema Vitoria & Valencia \\
\hline Inés Roig & Alicante \\
\hline Jaime Botella & Valencia \\
\hline Josefina Velert & Valencia \\
\hline Luis Salar & Valencia \\
\hline Maite Climent & Valencia \\
\hline Paz Sancho & Valencia \\
\hline Rosa Prats & Alicante \\
\hline Vicente Baixauli & Valencia \\
\hline
\end{tabular}

\section{Referencias bibliográficas}

1. Orden de 16 de abril de 2002, del Conseller de Sanidad por la que se crea el sistema de información farmacéutico Gaia: Gestor integral de la Prestación Farmacéutica de la Conselleria de Sanidad, se establecen los órganos de dirección del sistema, mantenimiento de las bases de datos, comités de apoyo y procedimientos de control de calidad. DOGV num. 4265, 15556-15569 [citado 15 feb 2015]. Disponible en: http://www.san.gva. es/documents/152919/183036/DOCUMENT01.pdf

2. Resolución de 4 de marzo de 2002, del Conseller de Sanidad, por la que se adecua el formato del documento oficial informatizado, Receta del Sistema Nacional de Salud de la Comunidad Valenciana editado por el Gestor Integral de la Prestación Farmacéutica GAIA. DOGV num. 4234, 11177-11183 [citado 15 feb 2015]. Disponible en: http://www.san.gva.es/documents/152919/183036/Norma2.pdf

3. Concierto entre la Conselleria de Sanitat y los Colegios Oficiales de Farmacéuticos de las provincias de Alicante, Castellón y Valencia. 2004 [citado 15 feb 2015] Disponible en: http://www.san.gva.es/docu- ments/152919/183036/22_web_concierto.pdf

4. De la Poza E, Barrachina I, Trillo JL, Usó R. Sistema de prescripción y dispensación electrónica en la Agencia Valenciana de Salud. El profesional de la Información [Internet]. 2011 [citado 16 feb 2015]. 20(3): 332-339. Disponible en: http://www.ciegs.upv.es/is/sistema_prescripcion_dispensacion_electronica_agencia_valenciana_salud.html

5. Convenio de colaboración entre la Agencia Valenciana de Salud de la Conselleria de Sanitat y los Colegios Oficiales de Farmacéuticos de las provincias de Alicante, Castellón y Valencia para el desarrollo de la "Atención Farmacéutica electrónica - dispensación electrónica" , en el marco de la Receta Electrónica de la Generalitat Valenciana. 2007 [citado 15 feb 2015]. Disponible en: http://www.san.gva. es/documents/152919/183036/23_ web_conveniodesarrollo.pdf

6. Documento anexo para la implantación de un modelo de dispensación y facturación en el sistema integrado de receta electrónica de la Conselleria de Sanitat de la Generalitat. 2008 [citado 16 feb 2015]. Disponible en: http://www.san.gva.es/documents/152919/168998/3convenioimplantacion.pdf

7. Brizuela L. Estudio de implantación de receta electrónica en España [Resumen]. Farmacéuticos Comunitarios [Internet]. 2014 [citado 15 feb 2015]. 6 (supl. 1). Disponible en: http://www. farmaceuticoscomunitarios.org/journal-article/estudio-implantacion-receta-electronica-espana

8. La receta electrónica termina de implantarse en septiembre. Redacción médica [Internet]. 2013 [citado 15 feb 2015]. Disponible en: http://www. redaccionmedica.com/autonomias/ valencia/la-receta-electronica-termina-de-implantarse-en-septiembre-8432

9. Costa D. La receta electrónica llegará en septiembre a toda la Comunidad. Diario Información [Internet]. 2013 [citado 15 feb 2015]. Disponible en: http:// www.diarioinformacion.com/alicante/2013/07/07/sanidad-fija-septiembre-extension-comunidad/1393039. html

10. Baixauli VJ, Prats R, Aparicio C, Colomer V. Causas de las incidencias por no conformidad en la dispensación de la receta electrónica. Farmacéuticos Comunitarios. 6(Suplemento 1).

11. Colomer V, Vañó A, Belda P. Análisis de las causas de no retirada de medicamentos por el paciente en farmacia comunitaria. Farmacéuticos Comunitarios. 6(Suplemento 1).

12. Prats R, Piera V, Borja C. Receta electrónica: ¿por qué rechaza el paciente su medicación prescrita?. Farmacéuticos Comunitarios. 6(Suplemento 1).

13. ADHERENCE TO LONG-TERM THERAPIES. Evidence for action. WHO 2003. Disponible en https://apps.who.int/iris/bitstream/handle/10665/42682/9241545992. pdf?sequence $=1$

14. Gabinete Sociología y Comunicación. Encuesta sobre adherencia terapéutica en España. Fundación Farmaindustria [monografía en internet]. Madrid: Sociología y comunicación; 2016. [citado 15/04/2019]. Disponible en: http:// www.farmaindustria.es/adherencia/ wp-content/uploads/sites/6/2016/11/ farmaindustria-plan-de-adherencia. pdf?v=2017

15. Radek Pelouch, Viktor Voříšek, Věra Furmanová, Miroslav Solař. The Assessment of Serum Drug Levels to Diagnose Non-Adherence in Stable Chronic Heart Failure Patients. Acta Medica (Hradec Králové) 2019; 62(2): QQQ-QQQ. doi:10.14712/1805 9694.2019.4

16. Otón T, Carmona L, Urruticoechea-Arana A, Calvo-Alén J, Arteaga MJ, Cea-Calvo L. Discordance between doctor and patient assessments and non-adherence to subcutaneous biological drugs. Rheumatol Int 2019; 39: 1077. doi:10.1007/s00296-01904304-w

17. Osterberg L, Blaschke $\mathrm{T}$ (2005) Adherence to medication. N Engl J Med 353(5):487-497. doi:10.1056/NEJMra 050100

18. Gadallah MA, Boulos DN, Gebrel A, Dewedar S, Morisky DE (2015) Assessment of rheumatoid arthritis patients' adherence to treatment. Am J Med Sci 349(2):151-156. doi:10.1097/ MAJ.0000000000000376

19. Conthe $\mathrm{P}, \mathrm{Má}$ quez Contreras E, Aliaga Pérez A, Barragán García B, Fernández de Cano Martín MN et al. Adherencia terapéutica en la enfermedad crónica: estado de la situación y perspectiva de futuro. Rev Clin Esp. 2014. doi:10.1016/j. rce.2014.03.008

20. Clemente JM, Font B, Lahoz R, Llauradó G, Gambús G, Grupo de investigadores del Estudio INERCIA Inercia clínica en pacientes con diabetes mellitus tipo 2 no insulinizados en tratamiento con hipoglucemiantes orales. Estudio INERCIA. Med Clin (Barc)., 142 (2014), pp. 478-484. doi:10.1016/j.medcli.2013.02.032

21. Piñeiro-Abad A, Mera-Gallego R, Andrés-Iglesias JC, Fornos-Pérez JA, Vérez-Cotelo N, Andrés-Rodríguez NF. Análisis del rechazo de dispensaciones en receta electrónica y la relación con el incumplimiento. Pharm Care Esp. 2018; 20(4): 247268. Disponible en: https://www. 
pharmcareesp.com/index.php/PharmaCARE/article/view/441/367

22. González López-Valcárcel B, Librero J, García-Sempere A, et al. Effect of cost sharing on adherence to evidencebased medications in patients with acute coronary syndrome. Heart 2017;103:1082-1088. doi:10.1136/heartjnl-2016-310610

23. Salar L, Solá N, Cámara R, Cosín A, Dago A, Gutiérrez P. Resumen de resultados del Programa D-VALOR . Farmacéuticos Comunitarios. 4(Suplemento 1).

24. R. Cámara, A. Cosín, A. Dago, P. Gutiérrez, L. Salar, N. Solá. Programa D-VALOR, análisis de registros de dispensación. Benzodiazepinas. Pharm Care Esp. 2013; 15(4): 140-6.

25. A. Dago, A. Cosin, P. Gutiérrez, R. Rica, L. Salar, N. Solá. Análisis de registros de dispensación en antiinflamatorios no esteroideos dentro del programa D-Valor. Pharm Care Esp. 2013; 15(2): 51-8.

26. Solá N, Cámara R, Cosín A, Dago A, Gutiérrez P, Salar L. Programa D-Valor: Evaluación de registros de dispensación de estatinas. Farmacéuticos Comunitarios. 2013 May 30; 5(2):65-68

27. Salar L, Solá N, Cámara R, Cosín A, Dago A, Gutiérrez P. Programa D-Valor: evaluación del servicio de dispensación de bifosfonatos. Farmacéuticos Comunitarios. 2014 Sep 30; 6(3):5-11. doi:10.5672/FC.2173-9218.(2014/ Vol6).003.02

28. López Díaz J, Alejandre Lázaro G, Redondo de Pedro S, Soto García M, López de Castro F y Rodríguez Alcalá FJ. ¿Comprenden los pacientes el tratamiento antibiótico prescrito? Aten Primaria 2001. 15 de octubre. 28 (6): 386-390. doi:10.1016/S02126567(01)70400-3

29. Elena Bernabé Muñoz, Macarena Flores Dorado, Fernando Martínez Martínez. Grado de conocimiento del antibiótico prescrito en pacientes ambulatorios. Aten Primaria. 2015;47(4):228-235. doi:10.1016/j. aprim.2014.04.014

30. Shivangini Singh, Sahana Devadasa Acharya, Ashwin Kamath, Sheetal D. Ullal, Rathnakar P. Urval. Health Lite- racy Status and Understanding of the Prescription Instructions in Diabetic Patients. Journal of Diabetes Research. Volume 2018, Article ID 4517243, 5 p. doi:10.1155/2018/4517243

31. Terry C. Davis, PhD; Michael S. Wolf, PhD, MPH; Pat F. Bass III, MD; Jason A. Thompson, BA; Hugh H. Tilson, MD, DrPH; Marolee Neuberger, MS; Ruth M. Parker, MD. Literacy and Misunderstanding Prescription Drug Labels. Ann Intern Med. 2006;145(12):887894. doi:10.7326/0003-4819-145-12200612190-00144

32. López García-Ramos L. La receta electrónica como método de medida del cumplimiento terapéutico en hipertensión arterial en atención primaria [Tesis doctoral]. Universidad de Sevilla. 2013.

33. Vazquez, Victoria, Chacón, Juán, Espejo, José, Faus, María José. Resultados del seguimiento farmacoterapéutico en una farmacia comunitaria. Pharmacy Practice [en línea] 2004, 2 (july-september): [Fecha de consulta: 5 de mayo de 2019] Disponible en: http://www.redalyc.org/ pdf/690/69020306.pdf 